\title{
„Die gute alte Zeit und das gute alte Wien gehören zueinander wie ein Paar Eheleute.“ (Heinrich Laube) Alt-Wien in der österreichischen Literatur von 1850 bis 1930: Die Stadt, die niemals war?
}

\author{
Marc Lacheny, Prof. an der Universität Lothringen (Metz)
}

Sobald die Literatur sich des Gegenstands „Stadt“ annimmt, um ihn in ein literarisches Thema zu verwandeln, wird die Stadt - hier Wien - zur Metapher ihrer selbst, ja zu einer Art Wunschvorstellung oder Idealisierung der wirklichen Stadt. Dies gilt insbesondere für die vielfältige österreichische Literatur von 1880 bis 1930, die zur Entstehung des Mythos „AltWien" beigetragen hat. Der vorliegende Aufsatz, der auf einer Auswahl an Texten aus der österreichischen Literatur und Publizistik des 19. und 20. Jahrhunderts (u.a. Spitzer, Kraus, Zweig, Hofmannsthal) beruht, verfolgt die Absicht, die Verbindungen und Interaktionen zwischen „Alt-,, und „Neu-Wien“" ans Licht zu bringen, aber auch die sich immer deutlicher erweiternde Kluft zum Vorschein zu bringen, die sich gerade in dieser Produktion zwischen dem Mythos „Alt-Wien“ als imaginärer Stadt bzw. als literarischer Konstruktion und „NeuWien" als wirklicher Stadt und - auch unheimlichem - Schmelztiegel der Modernität artikuliert. In einem solchen Zusammenhang erfüllen die utopischen Komponenten, die im Mythos Alt-Wien enthalten sind, vor allem folgende Funktionen: Flucht/Ausflucht, Sublimierung/Idealisierung, Schöpfung eines utopischen bequemen „Unorts“ (im Sinne von Marc Augé) mit stark konservativen Zügen, was Karl Kraus 1912 zu einer ironischen Bemerkung anregte: „Ich mu $\beta$ den Ästheten eine niederschmetternde Mitteilung machen: AltWien war einmal neu."

Die aktuelle Auseinandersetzung mit dem Raum (spatial oder topographical turn) knüpft an eine ältere Debatte an: Um und nach 1900 waren schon Georg Simmel und Walter Benjamin maßgebliche Denker und Theoretiker des Raums. Nicht unerwähnt können heute Positionen wie diejenigen von Michel de Certeau, Marc Augé, Karl Schlögel oder Martina Löw bleiben. Stadt erscheint in ihren Qualitäten als ein Gebautes, Genutztes, Erfahrenes, Dargestelltes, wobei Literatur immer wieder bestimmte Bilder von Stadt, also Stadtrepräsentationen, erzeugt.

Vom 25. November 2004 bis zum 28. März 2005 widmete das Wien Museum dem Thema „Alt-Wien. Die Stadt, die niemals war““ eine große Ausstellung. ${ }^{1}$ Von Anfang an lässt sich feststellen, dass „Alt-Wien“, ein mit Nostalgie und Melancholie gesättigter Begriff, der

\footnotetext{
Wolfgang Kos und Christian Rapp (Hg.), Alt-Wien. Die Stadt, die niemals war, 316. Sonderausstellung des Wien Museums (bis 2003 Historisches Museum der Stadt Wien), Wien Museum im Künstlerhaus. Wien: Czernin 2004; Arnold Klaffenböck (Hg.), Sehnsucht nach Alt-Wien. Texte zur Stadt, die niemals war. Wien: Czernin 2005.
} 
sich im Vormärz ${ }^{2}$ eingebürgert hat und besonders in den Romanen und Erzählungen an der Wende vom 19. zum 20. Jahrhundert wieder deutlich an Bedeutung gewinnt, als geistige Konstruktion, Mythos, utopische Projektion erscheint, die mit vielen Stereotypen verknüpft ist und deren Funktion vor allem darin besteht, die jetzigen Verfechter der Wiener Moderne bzw. des neuen Wien an die „Echtheit“ eines vergangenen und idealisierten Wien zu erinnern.

Seit dem 19. Jahrhundert haben sich viele österreichische Schriftsteller - in Gedichten und Elegien, vor allem aber in Romanen und Aufsätzen - mit dem Thema „Alt-Wien“ auseinandergesetzt: u.a. Auernheimer, Spitzer, Kraus, Friedell, Zweig, Schnitzler, Hofmannsthal, Aichinger, Bachmann. Alle diese Autoren, und viele weitere Vertreter der damaligen Unterhaltungsliteratur, haben dazu beigetragen, in der Behandlung des Themas einige Konstanten festzusetzen, die eben Alt-Wien seine Konturen als „Denkfigur“ (Wolfgang Kos), als Vorstellungskomplex, als imaginäre Stadt, ja als „Architektur der Psyche“, ${ }^{3}$ als Phantasma und Metapher der wirklichen Stadt verliehen haben. In diesem Kontext interessieren wir uns insbesondere für den diskursiv ständig hervorgehobenen Gegensatz zwischen Altem (als Vorbild) und Neuem (als Drohung und Gefahr), sowie für die Etablierung von Klischees, Stereotypen und Vereinfachungen, die zur literarischen Konstruktion von „Alt-Wien“ als imaginärer Stadt und Verbindung zwischen Realität und Phantasie beigetragen haben. Das Wort „Klischee“ ist hier nicht unbedingt negativ zu verstehen, denn auch Klischees speichern Wirklichkeit.

Der erste Teil dieser Ausführungen wird zu zeigen versuchen, wie Alt-Wien sich in der literarischen und publizistischen Produktion zwischen 1850 und 1930 als Mythos und als Utopie bzw. als „Unort“ konstituiert. Durch das verzerrende Prisma der Literatur verwandelt sich Wien nämlich in eine zeitlose und ewige Stadt, die alle Symptome einer ästhetischen Phantasmagorie aufweist. Der zweite Teil versucht die Verbindungen und Interaktionen zwischen „Alt-,, und „Neu-Wien“, aber auch die sich immer deutlicher erweiternde Kluft zum Vorschein zu bringen, die gerade in dieser Produktion zwischen dem Mythos „Alt-Wien“als imaginärer Stadt bzw. als literarischer Konstruktion - und „Neu-Wien“ - als wirklicher Stadt und (auch unheimlichem) Schmelztiegel der kulturellen und urbanen Modernität - zum Ausdruck kommt.

\footnotetext{
2 Vgl. Sándor Békési, „Alt-Wien oder Die Vergänglichkeit der Stadt. Zur Entstehung einer urbanen Erinnerungskultur vor 1848“. In: Alt-Wien. Die Stadt, die niemals war (wie Anm. 1), S. 29-38, hier: S. 38.

3 Carsten Lange, Architekturen der Psyche: Raumdarstellung in der Literatur der Romantik. Würzburg: Königshausen \& Neumann 2007, S. 7.
} 


\section{Alt-Wien: Konstruktion eines Mythos zwischen Sehnsucht, Utopie und urbaner Phantasmagorie}

Um 1900 wird die ,alte“ Stadt Wien zu einem bedeutenden Topos in der österreichischen Trivial- und Unterhaltungsliteratur: ${ }^{4}$

Nichts ist in den letzten Jahren im Roman beliebter geworden als das Alt-Wiener Genre. Halb und halb getragen von einem Modezug, zur anderen Hälfte aber doch von einem tieferen Gefühle geleitet, hat sich die jüngste Wiener Generation mit innigem Gefühl und wirklicher Sehnsucht in jene verwichenen Tage vertieft. Je mehr das alte Wien aus dem Straßenbild entschwand, um so lebhafter, dringender, innerlicher wurde das Verlangen, uns in die große Wendezeit der österreichischen Kunst und Kultur zurückzuversetzen. ${ }^{5}$

Eben zu dieser Zeit verschärfen sich die Kontroversen über das „neue“ Wien sowie allgemeiner - über die Lebensbedingungen in der modernen Großstadt. Dabei wurde Wien zu einem besonders umstrittenen Topos im Diskurs über die Beziehungen zwischen Urbanität und Modernität.

Konzentriert man sich auf berühmte Vertreter der Wiener Moderne wie Hofmannsthal und Schnitzler oder auf populäre Gattungen wie das literarische Feuilleton (Ferdinand Kürnberger, Ludwig Speidel, Daniel Spitzer, Friedrich Kilian Schlögl) oder die Unterhaltungsliteratur, steht ein großer Teil der literarischen Produktion aus Österreich um die Wende zum 20. Jahrhundert im Zeichen der Melancholie, der Nostalgie, der Sehnsucht nach längst abgeschlossenen und idealisierten Epochen, die der bedrohlichen Gegenwart entgegengesetzt werden. Tiefe Skepsis gegenüber den Veränderungen des urbanen Körpers äußert etwa - unter vielen anderen - Adalbert Stifter in Der Tandelmarkt, wo er die Verdrängung mannigfaltiger Häuser und kleinteiliger Strukturen durch die neue Architektur des mehrstöckigen Mietshauses mit strenger Kubatur bedauert. Um 1900 wird Alt-Wien mehr denn je zu einem Reservoir ästhetisch-moralischer Werte und zu einem Sinnbild für als verloren empfundene soziopolitische Inhalte. ${ }^{6}$ Diese Entwicklung verschärft sich mit dem Zerfall des Habsburger-Reiches und nach 1918, ,als der pulsierenden Metropole durch die

\footnotetext{
$4 \quad$ Vgl. Arnold Klaffenböck, „Ferdinand Raimund und das ,Alt-Wiener Antlitz‘. Bilder urbaner Identität in der Unterhaltungsliteratur zwischen 1900 und 1945“. Nestroyana, Band 26 (2006), Heft 3-4, S. 148-164, hier: S. 149 .

Rudolf Holzer, „Aus Wiens klassischer Zeit“. Wiener Abendpost. Beilage zur Wiener Zeitung, 24. Oktober 1912.

6 Vgl. Klaffenböck, „Literarische Positionen zu Alt-Wien“. In: Alt-Wien. Die Stadt, die niemals war (wie Anm. 1), S. 217-227, hier: S. 219.
} 
plötzliche Randlage, durch Abwanderung und (Welt-)Wirtschaftskrise der Abstieg in die Bedeutungslosigkeit und Provinzialität drohte. “7

Die (Unterhaltungs-)Literatur reagierte auf diese historische Situation mit makellosen Ersatz- oder Gegenwelten, wobei die Idealisierung einstiger Harmonie vor allem als Abwehrschild gegenüber der als bedrohend empfundenen Gegenwart und der realen Erfahrung von Zerstörbarkeit fungiert: Dynamik erscheint gefährlich, Stillstand (bzw. Statik) aber positiv, weil er Sicherheit bietet; die Ablehnung der Stadterneuerung verbindet sich mit rückwärts gewandten Utopien zu einem deutlichen Gegensatz zur Moderne. Gleichgültig, ob die verschiedenen Autoren die Handlung ihrer Romane im Barock des Prinzen Eugen, in der Ära Maria Theresias, im Vormärz oder im Zeitalter Kaiser Franz Josephs I. ansiedeln: Allen erscheint Alt-Wien als eine makellose Rückprojektion und als ein zutiefst verinnerlichtes Ideal, die sich von der Wirklichkeit der neuen Stadt nicht besudeln lassen. Wir haben es hier mit einer literarischen Darstellung zu tun, die den Mythos des ewigen und unsterblichen Wien andauernd fortbestehen lässt. Eines der konkreten stilistischen Kennzeichen für diese massive Sehnsucht nach einer erträumten Vergangenheit ist die Omnipräsenz einer stilistischen Sentimentalität, die nicht selten mit einer Personifizierung oder Vermenschlichung Alt-Wiens einhergeht, ${ }^{8}$ wobei Lebensgeschichten historischer (literarischer, musikalischer, künstlerischer) Persönlichkeiten ausgewählt werden, die in diesem Zusammenhang geeignet scheinen, populär oder identitätsstiftend (bzw. -bewahrend) zu wirken: Der Kritiker und Feuilletonist Raoul Auernheimer (1876-1948) kommt etwa auf sein Verlangen zu sprechen, „,von Alt-Wien zu träumen, der im Meer der Zeiten so tief versunkenen Stadt“ ${ }^{9}{ }^{9}$ Hier lässt sich der sentimentale und nostalgische Kult kaum von der Suche nach einer geistigen Heimat und Zugehörigkeit unterscheiden, wobei individuelle Züge, wie die des Wiener Volksdramatikers Ferdinand Raimunds, auf den gemeinsamen urbanen Körper Alt-Wiens direkt projiziert oder übertragen werden. ${ }^{10}$

Zwei Hauptelemente, die sich in der Literatur wiederholen und ergänzen, lassen die urbane Phantasmagorie „Alt-Wien“ entstehen.

Erstens betrachten die Autoren der untersuchten Romane Wiens Topographie als eine idyllische Stadtlandschaft, in der die Natur (im Zusammenhang mit einer scharfen

\footnotetext{
$7 \quad$ Vgl. Klaffenböck (wie Anm. 4), S. 150.

8 Zur Humanisierung des Ortes und deren Bedeutung für die „Lektüre“ der Stadt vgl. Richard Sennett, Civitas. Die Großstadt und die Kultur des Unterschieds. Frankfurt-am-Main: Fischer 1991, insb. S. 245.

$9 \quad$ Raoul Auernheimer, „Alt-Wien im Spiegel“. In: R. A., Das ältere Wien. Bilder und Schatten. Leipzig: Wien Tal 1920, S. 53-62, hier: S. 54.

10 Dies ist zum Beispiel in Ferdinand Raimunds letzte Liebe von Pankraz Schuk der Fall. Diese Sichtweise des urbanen Körpers (Stadt als Organismus) ist u.a. auch bei Stifter zu finden.
} 
Zivilisationskritik) überall erreich- und abrufbar ist: Untrennbar mit der Architektur ist die Stadt mit der sie umgebenden und durchdringenden Natur verbunden (Heurigen in den Wiener Vororten, Dörfer in der Umgebung, Wienerwald). Hier fällt insbesondere der Kult der frühlingshaft-heiteren Stadt und des Frohsinns im Kontakt mit der Natur auf, wobei „AltWien“ als Locus amoenus wirkt: „Die Stadt wird als Paradies ausgewiesen, das im Einklang mit den überirdischen Mächten stehe und von der Schöpfung bevorzugt wäre.“11 Diesen Eindruck vermittelt etwa Pankraz Schuk (1877-1951) mit seinem historischen Roman Ferdinand Raimunds letzte Liebe (1913), in dem Alt-Wien - fast wortwörtlich - zu einem Paradies auf Erden erhoben wird:

Das Himmelgewölbe, das wie eine riesenmächtige, gläserne Glocke über dem Häusergewirr von Wien hängt, ist von einem Dufte und einer Klarheit, da $\beta$ eines meint, sein Blick müsse es durchdringen und Gottvater ersehen können auf seinem Weltenthron, und die Luft ist so wohlig, als hätten die Engerln sie vorerst erwärmt, ehe sie sie hinabgeblasen über alle Welt. ${ }^{12}$

Sowohl in Otto Hausers Alt-Wien (1911) als auch in Eduard Paul Danszkys Roman „Da leg' ich meinen Hobel hin... “ (1939) - ein Zitat aus Ferdinand Raimunds berühmtem „Hobellied“ (Der Verschwender) - verliert Wien die gute Laune nicht. Diese dominiert so sehr, dass bei Hauser die Napoleonischen Kriege und bei Danszky der Wiener Kongress keinesfalls als historische Wendepunkte, sondern eher als vergängliche Ereignisse dargestellt werden.

Auf dem Land, etwa im Wienerwald, entziehen sich die Protagonisten den Zwängen des städtischen Lebens und erleben Glück, seelischen Frieden, Freiheit und Ruhe - so etwa im Roman Rappelkopf (1922) von Fritz Stüber-Gunther, wo Raimund zur Ruhe kommt und Augenblicke der Wonne erlebt:

Das war, wenn er durch die romantischen Täler der Brühl, durch die Forste des Anninger streifte, wenn er zu den alten Schlössern des Badener Tales emporstieg und den Steinen, den Felsen, den Waldriesen schwärmerisch den Namen Antonia zurief; wenn er auf blumigen Wiesen lagerte und oben am blauen Himmel Engelsbilder sich malte, die ihm freundlich zunickten, und deren herrlichstes, alle anderen überstrahlendes das Antlitz seines Mädchens trug, wenn der laue Wind ihm ihre süße Stimme zuzuwehen schien, wenn er die Augen schlo $\beta$ und sich mit aller Kraft seiner Phantasie einbildete, die Ferne weile neben ihm... ${ }^{13}$

$11 \quad$ Vgl. Klaffenböck (wie Anm. 4), S. 152.

12 Pankraz Schuk, Ferdinand Raimunds letzte Liebe. Wiener Roman aus den Zwanzigerjahren des 19. Jahrhunderts. Wien: Hesse \& Becker 1913, S. 3.

Fritz Stüber-Gunther, Rappelkopf. Roman. Wien: Wila-Verlag K. Fiedler 1922, S. 311 f. 
Es geht hier keinesfalls darum, eine unmittelbare Naturlandschaft wiederzugeben oder einzufangen, sondern auf eben diese Landschaft psychologische, literarisch gestaltete, symbolische Elemente zu projizieren, so dass eine Seelenlandschaft oder eine Kartographie der Imagination der vertretenen Autoren zu Tage tritt.

Zweitens frappiert das Lob der Wiener Musik, insbesondere am Beispiel von Komponisten wie Beethoven, Schubert, ${ }^{14}$ Josef Lanner oder Johann Strauß sen., die weit höher als die neuen Operetten eingeschätzt werden: Z.B. widmet 1926 Joseph August Lux (1871-1947) - von dem auch ähnliche Schilderungen von Schubert, Grillparzer und Liszt stammen - Beethoven einen biographischen Roman mit dem Titel Beethovens unsterbliche Geliebte, und Otto Hauser nimmt sich in seinem Roman Alt-Wien (1911) die Lebenswelt Ludwig van Beethovens zum Vorbild. Hausers Alt-Wien sowie Hans Harts Liebesmusik (1910), wo die Schicksale der Protagonisten mit den Lebenswegen von Künstlern wie Franz Schubert oder Moritz von Schwind zusammenlaufen, entführen in eine frühlingshaft-heitere Stadt, in der alles in musikalische Schwingungen gerät und wo alle glücklich sind. Genauso fällt der Kult gewisser Theaterautoren und Schauspieler auf, die mit einer zeitlosen Wiener Vergangenheit verbunden werden - insbesondere Franz Grillparzer, Ferdinand Raimund, ${ }^{15}$ Adalbert Stifter, die schon zu Lebzeiten sehr populäre Schauspielerin und Sängerin Therese Krones (1801-1830), eine Schlüsselfigur des Alt-Wien-Komplexes auch in der Operette,

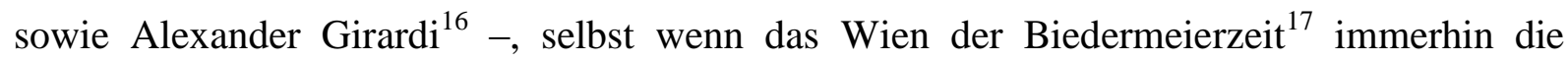
Epoche bleibt, auf die die meisten Autoren zurückblicken. In dem Roman Hinter der lachenden Maske (1939) von Else von Hollander-Lossow (1884-?), einer Lebensbeschreibung von Ferdinand Raimund, sieht Therese Krones' Vater die alte Stadt Wien als die „Sehnsucht

$14 \quad$ Zu diesem Thema vgl. Ulrike Spring, „Der Himmel über Wien. Franz Schubert, sein Körper und AltWien“. In: Alt-Wien. Die Stadt, die niemals war (wie Anm. 1), S. 151-158, sowie „Raum 6: Schwammerls Wien. Schubert als personifiziertes Biedermeier“, S. 423-439. Diese Seiten zeigen, dass Franz Schubert als ideale Projektionsfläche diente und zu einer zentralen Symbolfigur des alten Wien wurde, wobei Schubert sowohl als Komponist als auch als fiktive Gestalt gefeiert wurde: Er war auf Gemälden, auf Ansichtskarten und auf Taschentüchern omnipräsent, und sogar Schubert-Büsten aus Seife waren am Markt. Ein wichtiger Schritt vom Schubert-Kult zum Schubert-Kitsch lässt sich auf das Jahr 1911 datieren, als der Roman Schwammerl von Rudolf H. Bartsch erschien, der zum Bestseller wurde.

15 Im 20. Jahrhundert insistieren Pankraz Schuk, Fritz Stüber-Gunther, Karl Hans Strobl, Hertha Pauli, Else von Hollander-Lossow und Eduard Paul Danszky auf der engen Verbindung zwischen Raimund und AltWien, wobei Raimund immer wieder - wie die biedermeierliche Stadt selbst - als „disponible Projektionsfigur“ (Klaffenböck, wie Anm. 4, S. 152) benutzt wird.

16 Vgl. hierzu Marion Linhardt, „Ein ,neuer“ Raimund?! Alexander Girardis Rolle für die Alt-WienRezeption um 1900“. Nestroyana, Band 26 (2006), Heft 3-4, S. 165-184. In seinen Raimund-Rollen galt Girardi als die Verkörperung Alt-Wiens, und in diesem Kontext wurde er mit einer traditionellen sozialen Ordnung identifiziert, die im biedermeierlichen Kult der Familie ihren Niederschlag findet. In seinen Strauß- und Millöcker-Partien galt Girardi genauso als einer der bedeutendsten Retter der „guten alten“ Wiener Operette.

17 Das Wort Biedermeier wird hier nicht als ein Begriff verstanden, der sehr genau eine Epoche umfasst, sondern eher als der nostalgische und idealisierte Blick, der an der Wende zum 20. Jahrhundert auf die Jahre um den Wiener Kongress geworfen wurde. Dabei wurde das Biedermeier zu einer zwar idyllischen, aber historisch ungenauen Epoche verklärt. 
seines Lebens" ${ }^{\text {(18 }}$ an, genauso wie der Dichter Friedrich Sauter aus Eduard Paul Danszkys Roman Er war in Wien verliebt (1944). Ein Jahrhundert früher, in Aussicht und Betrachtungen von der Spitze des St. Stephansthurmes (1844), präsentierte Adalbert Stifter Wien schon als ein wohl geordnetes und sinnvolles urbanes Netzwerk, das er mit Begriffen aus der Geometrie und Mathematik zu erfassen versuchte.

Die Natur, die Jahreszeiten und die Welt der Musik und der Literatur - als besonders wirkungsmächtige Klischees - erscheinen hier als Symbole für Beständigkeit, Dauer und Ewigkeit, jenseits von Zeit und Raum, als bevorzugte Mittel, die Zeit aufzuhalten: „Die literarische Fiktion suggeriert, dass Alt-Wien trotz der Zeitenläufe überdauert habe und noch bestehe.“19 Es geht nämlich darum, in Wien eine Art von verschwundenem Genius loci wiederzufinden und aus Alt-Wien ein irdisches Paradies, einen mit Lebensfreude verbundenen Raum, das nostalgische Refugium einer ewigen ,guten alten Zeit“" zu machen, die mit den negativen Erfahrungen mit der modernen Großstadt kontrastiert. Angesichts der Realität des verschwindenden alten Wien, bedingt durch die massiven städtebaulichen Veränderungen im letzten Drittel des 19. Jahrhunderts (allein zwischen 1850 und 1900, also in den Jahren, in denen „Alt-Wien“ wirklich populär wurde, hat man 50 Prozent aller Häuser der Innenstadt demoliert und durch Neubauten ersetzt), versucht die Literatur diese historische Entwicklung und die Realität des Untergangs zu korrigieren. Mit anderen Worten: Die Autoren, die Alt-Wien besingen, versuchen innerhalb des durch die Literatur erzeugten urbanen Unorts den Verlauf einer geschichtlichen Entwicklung zu verhindern, die als Gefahr empfunden wird. Die literarische Produktion zu Alt-Wien ersetzt die Stadt als realen und widersprüchlichen Ort durch einen nivellierten, enthistorisierten, idealisierten kulturellen Raum, der weder der Gegenwart noch der Vergangenheit des Biedermeiers entspricht: „Zum Mythos ,Alt-Wien` gehört das (Trug-)Bild eines idyllischen, gemütlichen, liebenswürdigen Biedermeiers. Tatsächlich waren die vormärzlichen Zeiten unruhig und chaotisch, das Veränderungstempo war hoch, der Straßenverkehr hektisch, die hygienischen Verhältnisse waren katastrophal. Und das Stadtbild wurde rücksichtslos verändert: Vertraute Häuserzeilen wurden demoliert, um vielstöckigen Zinsburgen Platz zu machen. “20

\footnotetext{
18 Else von Hollander-Lossow, Hinter der lachenden Maske. Ein Alt-Wiener Roman. Hannover: Sponholtz 1939, S. 21.

Arnold Klaffenböck, ,„In jedem Treppenwinkel blüht hier ein Roman. ' Diskurse von Alt-Neu-Wien in der Unterhaltungsliteratur 1860-1938“. In: Mythos Alt-Wien. Spannungsfelder urbaner Identitäten. Hrsg. von Monika Sommer und Heidemarie Uhl. Innsbruck: StudienVerlag 2009, S. 121-149, hier: S. 127.

20 Wolfgang Kos, „Alt-Wien“ ist eine Denkfigur - Zur Einleitung“. In: Arnold Klaffenböck (Hg.), Sehnsucht nach Alt-Wien. Texte zur Stadt, die niemals war (wie Anm. 1), S. 8-11, hier: S. 9.
} 
Viele Autoren suchen folglich eine Flucht in eine ,,andere Welt“, in einen literarischen Unort, subsumiert im Bild eines metaphysischen und unzerstörbaren Wien, das überall und nirgends ist und als letztes Bollwerk gegen die Gefahren und Schattenseiten der Modernität fungiert. Hugo von Hofmannsthal macht z.B. aus Alt-Wien das Paradigma zur Bewältigung einer Gegenwart, die zugleich als unbefriedigend und bedrückend, ja sogar als unheimlich empfunden wird. ${ }^{21}$ Wie bei anderen Autoren konzentriert sich sein selektiver Blick ausschließlich auf die Phasen, in denen Wien kulturelle Höhepunkte erreicht hat: vor allem auf die Barockzeit Prinz Eugens, aber auch auf die Zeit Kaiserin Maria Theresias, dann auf den Josephinismus, die in den Augen des Dichters synonym für Initiativen und bedeutende Errungenschaften im Bereich der Dichtung, des Theaters und der Musik stehen - dies ist auch der Grund, warum der erbarmungslose Karl Kraus Hofmannsthals Rückblick auf die Vergangenheit mit einer besonders sarkastischen Bemerkung über seine „Habsburgerlippe“222 ins Lächerliche zog. Schließlich besitzt Hofmannsthals Konzeption von Alt-Wien eine ethische Dimension, die sich in der Berufung auf geistige bzw. dichterische Autoritäten wie Franz Grillparzer oder Ferdinand Raimund widerspiegelt. Hofmannsthals Alt-Wien-Lob wirkt kaum verwunderlich, da der Autor sich bekanntlich als Hüter und Erneuerer einer solchen Vergangenheit betrachtete. Sehr ähnlich erinnert auch Stefan Zweig an das „gute alte“ Wien der Biedermeierzeit und versucht im brasilianischen Exil das Wien seiner Kindheit in Die Welt von Gestern vor dem Vergessen zu bewahren:

Wenn ich zu Ihnen über das Wien von Gestern spreche, soll dies kein Nekrolog, keine oraison funèbre sein. Wir haben Wien in unseren Herzen noch nicht begraben, wir weigern uns zu glauben, da $\beta$ zeitwillige Unterordnung gleichbedeutend ist mit völliger Unterwerfung. Ich denke an Wien, so wie Sie an Brüder, an Freunde denken, die jetzt an der Front sind. Sie haben mit ihnen Ihre Kindheit verbracht, Sie haben Jahre mit ihnen gelebt, Sie danken ihnen glückliche, gemeinsame Stunden. Nun sind sie fern von Ihnen und Sie wissen sie in Gefahr, ohne ihnen beistehen, ohne diese Gefahr teilen zu können. Gerade in solchen Stunden erzwungener Ferne fühlt man sich den Nächsten am meisten verbunden. So will ich zu Ihnen von Wien sprechen, meiner Vaterstadt und einer der Hauptstädte unserer gemeinsamen europäischen Kultur. [... $]^{23}$

Auch in der Unterhaltungsliteratur erkennen Joseph August Lux (Das alte gemütliche Wien. Ein Buch von heiterer Lebenskunst, 1922), Hermine Cloeter (Geist und Geister aus dem alten Wien. Bilder und Gestalten, 1922) oder Ann Tizia Leitich (Verklungenes Wien, 1942) in

\footnotetext{
21 Vgl. z. B. Germanica, 43 (2008): Patrick Bergeron, „La marche à la mort: Vienne et Prague crépusculaires chez Hofmannsthal et Leppin“, S. 41-51, und Audrey Giboux, „Vienne selon Hofmannsthal: un adieu, entre critique et nostalgie“, S. 53-63.

22 Karl Kraus, „Aus der Barockzeit““. Die Fackel, 697-705 (1925), S. 81.

23 Stefan Zweig, „Das Wien von Gestern“ (1940). In: Arnold Klaffenböck (Hg.), Sehnsucht nach AltWien. Texte zur Stadt, die niemals war (wie Anm. 1), S. 151-158, hier: S. 151.
} 
einem zeitlosen Wien ein imaginäres Fluchtmittel aus der Gegenwart sowie eine besonders angemessene Gelegenheit, die Konsequenzen des verlorenen Krieges und des Wert- und Heimatverlustes zu überwinden. ${ }^{24}$ Etwa bei Hermine Cloeter (1879-1970), die viele kulturgeschichtliche Aufsätze über das alte Wien verfasst hat, werden Exkursionen durch ein imaginäres Alt-Wien unternommen: Die Stadt von einst wird mittels Fantasie und Erinnerung heraufbeschworen. Bei solchen Autoren wird Alt-Wien mit positiven moralischen, ästhetischen und soziopolitischen Tugenden und Werten assoziiert, deren Hauptfunktion darin besteht, in einer gemeinsamen Vergangenheit eine imaginäre kollektive Wiener Identität (wieder) zu finden.

Diese literarische Suche nach einer - ewigen und unberührten - geistigen und moralischen Heimat in „Alt-Wien“ spiegelt sich übrigens auch in anderen bedeutenden künstlerischen Ausdrucksformen wie dem Wienerlied. ${ }^{25}$ Wie in der Literatur zu Alt-Wien wird Wien darin allgemein als fiktives Kollektivum lobgepriesen, etwa in der Form des „UrWieners“, des ,alten Fiakers“ oder des ,alten Drahrers“, des ,goldenen“ Wienerherzens, kurz: der Stadt selbst als eines irdischen Paradieses und Ortes des Lebensgenusses (Wein, Weib und Gesang): „Der Weaner geht net unter“ (Anonym), „Die Weaner Gemütlichkeit stirbt niemals aus“ und „So warn 's uns're Alten g'wohnt!““ (beide von Carl Lorenz) „Da war's in Wien a Freud ein Wiener z' sein“ (Franz Schiferl), „Herrlich war 's in alten Zeiten“ (Eduard Merkt und Johann Sioly), „Erinnerung an Alt-Wien“ (Josef Hornig und Ferdinand Leicht) lauten die Titel nur einiger Lieder, die sich mit diesen obsessiven Themen befassen. Das Wienerlied wird dabei zu einer ganz besonderen Form des Psychogramms einer urbanen Mentalität, deren Grundzüge über zwei Jahrhunderte im Wesentlichen unverändert zu bleiben scheinen. Das Paradebeispiel für diesen Kult des Weins im Wienerlied verdanken wir Joachim Perinet (1763-1816): „Wer niemals einen Rausch hat g'habt, / Der ist ein schlechter Mann.“ In der wienerischen literarischen Tradition bilden Alkohol und Komik übrigens ein untrennbares Paar. ${ }^{26}$ Durchaus ähnlich verhält es sich in der Operette um $1900,{ }^{27}$ die im Zeichen einer

$24 \quad$ Vgl. zum Beispiel Ann Tizia Leitich, Wiener Biedermeier. Kultur, Kunst und Leben der alten Kaiserstadt vom Wiener Kongreß bis zum Sturmjahr 1848. Bielefeld, Leipzig: Velhagen \& Klafing 1941.

${ }_{25}$ Vgl. Eva Maria Hois und Ernst Weber, „,... doch die Zeiten sind dahin... !‘ Alt-Wien im Wienerlied“. In: Alt-Wien. Die Stadt, die niemals war (wie Anm. 1), S. 134-141, hier: S. 134.

26 Vgl. Gerald Stieg, „Alkohol auf dem Theater und im Lied von Mozart bis Qualtinger“. Nestroyana, Band 24 (2004), Heft 3-4, S. 134-142, hier: S. 135.

27 Vgl. Moritz Csáky, Ideologie der Operette und Wiener Moderne. Ein kulturhistorischer Essay zur österreichischen Identität. Wien, Köln, Weimar: Böhlau 1996, sowie Christian Glanz, „Himmelblaue Zeit. AltWien in der Operette“. In: Alt-Wien. Die Stadt, die niemals war (wie Anm. 1), S. 228-234, etwa S. 230-231: „Mit der aus dem musikalischen Nachlass des verstorbenen Komponisten von Adolf Müller jun. geschickt zusammengestellten Strauß-Operette Wiener Blut (Schauplatz ist das Wien zur Zeit des Wiener Kongresses, Wiener Volkstypen nehmen eine dramaturgisch betonte Rolle ein) beginnt 1899 eine Phase der Wiener Operettengeschichte, in der Alt-Wiener Sujets und Zusammenhänge besonders häufig werden. Heinrich Reinhardts Das süße Mädel (1901), Joseph Hellmesbergers Veilchenmädel (1904), Ernst Reiterers Strau $\beta$ 
idealen Identifizierung mit dem Wiener Genius loci (Musik und Theater) steht. Solche Nachbargenres erscheinen als Varianten einer urbanen Mentalität, deren Besonderheit in dem Widerstand gegen Dynamik, Veränderung und Modernität zu suchen ist. Außerdem bekommt „Alt-Wien“ nicht selten einen ideologischen Anstrich, der gelegentlich eine nationalistische Form annehmen kann, wie beim Schriftsteller, Theaterleiter und deutschnationalen Parlamentarier Adam Müller-Guttenbrunn (1852-1923), der nicht nur seine Alt-Wiener Wanderungen und Schilderungen (1915) publizierte, sondern auch zahlreiche Vorträge zur Wiener Geschichte hielt. Erneut erscheint Alt-Wien keinesfalls als eine Realität, die sich historisch erfassen lässt, sondern als ein Vorstellungskomplex, als die literarische Konstruktion eines Unorts, weit weg von der „realen“ Stadt. Alt-Wien wird also zu einem utopischen Raum, der zumindest in der Literatur weiterexistiert, auch wenn es ihn physisch eigentlich nicht mehr gibt. Das Gleiche gilt für das biedermeierliche Wien, das nie so ruhig und gemütlich war wie das Nachbild jener Epoche es suggeriert: Hierfür liefern die Werke von Franz Grillparzer, Ferdinand Raimund oder Johann Nestroy griffige Beispiele.

Die urbane Utopie, mit der der Leser der Literatur über Alt-Wien konfrontiert ist, entsteht also aus zwei Faktoren: Zuerst aus der quasi systematischen Absenz jeglicher genauen Zeitangabe (abgesehen von den wiederholten Anspielungen auf eine idealisierte Biedermeierzeit), was den Mythos einer zeitlosen Stadt namens „Alt-Wien“ bestätigt, ${ }^{28}$ dann aus ständigen Vereinfachungen, die mit der Komplexität der „modernen“ Stadt kontrastieren, welche - auf Grund ihrer sozioökonomischen Beschleunigung und der massiven Veränderungen ihrer Architektur (insbesondere in den letzten dreißig Jahren des 19. Jahrhunderts) - als eine immer deutlichere Bedrohung für den Alt-Wien-Mythos geschildert wird. Die Unterhaltungsromane entwerfen ein Bild Wiens, das mit den Problemen der wirklichen Stadt kaum zu tun hat, und perpetuieren stereotype und tröstliche Bilder aus der Vergangenheit, die im Gegensatz stehen zu den Schreckbildern und Ängsten, welche aus den technischen, wirtschaftlichen, politischen oder sozialen Veränderungen der „modernen“ Metropole entstanden sind.

Auswertung Gräfin Pepi (1902) seien hier ebenso erwähnt wie Leo Falls Singspiel Brüderlein fein (1909), Leo Aschers Operetten Hoheit tanzt Walzer (1912), Vindobona, du herrliche Stadt! (1910) und schließlich Emil Sterns Lanner-Bearbeitung Alt-Wien (1911).“

28 Vgl. auch Klaffenböck (wie Anm. 19), insb. S. 135. 


\section{Alt- vs Neu-Wien: Grenzen und Zerstörung eines Unorts?}

Es versteht sich von selbst, dass der unendliche Mythos „Alt-Wien“ äußerst ambivalent ist und sozusagen in sich selbst seine Grenzen enthält.

Die Literatur über Alt-Wien beruht nämlich grundsätzlich auf einer selbstreferenziellen und apologetischen Dimension, die einen kollektiven Wiener Narzissmus spiegelt, welcher weit über die Individualität der Persönlichkeiten hinausgeht, die als Symbol für die Größe Alt-Wiens gewählt wurden. Es geht um Texte, die vor allem von Wienern an Wiener verfasst wurden - nach dem Vorbild des „Mir san mir“, dessen Gefahren Karl Kraus in den Letzten Tagen der Menschheit erkannt hat:
Aus Tod wird Tanz, aus $\mathrm{Ha} \beta$ wird Gspa $\beta$, aus Not wird Pflanz, was is denn das? Is alles stier, is's einerlei, denn mir san mir und a dabei.
Ein guter Christ sagt: Kinder bet's, und Henker ist man nur aus Hetz. ${ }^{29}$

Wie Arnold Klaffenböck es am Beispiel von Fritz Stüber-Gunthers Roman Rappelkopf (1922) gezeigt hat, wirkt der „Blick auf Wien [...] nicht nur bewusstseinserweiternd, sondern ist zugleich einengend, weil sich die Sehnsucht schließlich doch nur auf diese Stadt konzentriert. Die Figuren kommen aus diesem Dunstkreis nicht heraus, die Introvertiertheit und Selbstgenügsamkeit Alt-Wiens halten sie gefangen. ${ }^{\text {‘30 }}$ Mit anderen Worten: Einer so affirmativen literarischen Produktion fehlt die kritische Basis, die es ihr erlauben würde, den zutiefst narzisstischen Aspekt zu nuancieren, der ihr zu Grunde liegt. In der Tat bietet AltWien perfekte Lebensmodelle, die sich von den Widersprüchen der realen Stadt fundamental unterscheiden: Wir haben es vielmehr mit einer ästhetischen Konstruktion zu tun, die zu einer Zeit der unvermeidlichen Veränderungen und des Übergangs zur Modernität ein tiefes Bedürfnis nach Nostalgie, Utopie und Beschwichtigung befriedigen soll. Hinter der

\footnotetext{
$29 \quad$ Karl Kraus, Die letzten Tage der Menschheit, V, 55 („Das österreichische Antlitz“). In: K. K., Schriften (Band 10). Hrsg. von Christian Wagenknecht. Frankfurt am Main: Suhrkamp 1986, S. 723.

$30 \quad$ Klaffenböck (wie Anm. 4), S. 159.
} 
scheinbaren Harmonie werden aber bald Schmerz und Bruchmomente deutlich. Die Utopie „Alt-Wien“ birgt in sich selbst die Dystopie.

Eben in diesem Kontext gewinnen der diskursive Gegensatz zwischen den Verfechtern des Alt-Wien-Modells und den Anhängern „Neu-Wiens“ (den „Modernisten“) sowie die konstruierte Kluft zwischen Alt- und Neu-Wien schon vor 1900 an Bedeutung. Schriftsteller wie Franz Gräffer (1785-1852), bei dem sich das schöne Wort „Stadtschmerz“ findet, haben schon um 1850 jenes Vokabular der klagenden Stadterinnerung entwickelt, das viele Jahrzehnte den dominierenden Diskurs über Alt-Wien charakterisieren sollte: „Ich war Zeuge davon. Ein unnennbar wehmütiges Gefühl hatte mich ergriffen und nagt noch an mir.“"31 Die neuen Häuser galten Gräffer als „kahl, monoton, flach“ und waren in seinen Augen von „,zinserträgrische[r] Ökonomie“ geprägt:

Wehe, wehe! Und nun suchen wir euer Konterfei als unsern einzigen Trost. Denn die neuen, auf euren allerletzten Spuren, stolzen, himmelanragenden neuen Häuser! - wir mögen sie nicht; sie sind uns, möchte ich sagen, nicht legitim genug; wir können sie nicht leiden, wir mögen sie nicht umsonst! Nie werden wir uns mit ihnen befreunden! Die kahlen, flachen, monotonen Dinger, ohne Höfe, ohne Räume, ohne Licht und Luft, mit ihrer egoistischen Enge und ihrer filzigen, zinserträgrischen Ökonomie. Die ganze gemütliche Wohnlichkeit, die ganze heimische Magie, die ganze alterliche Romantik sind dahin, dahin! Noch einmal: Wehe, wehe, und noch verschiedene Wehe! ${ }^{32}$

Bei Gräffer wie bei Stifter wird eine neue Erfahrung der Vergänglichkeit und des Identitätsverlusts spürbar, die mit der Zerstörung der alten urbanen Lebenswelt verbunden ist. Dieser Wandel des urbanen Körpers wird im selben Zeitraum aber auch von anderen Autoren - wie etwa Heinrich Joseph Adami (1807-1895) - positiv und fortschrittlich beurteilt:

Indessen Wien wird schöner und prachtvoller mit jedem Tage. Die unansehnlichen Häuser verschwinden, stolze Paläste nehmen ihre Stelle ein und das halb mittelalterliche Gepräge, welches die Stadt noch vor einem oder zwei Jahrzehnten dort und da hatte, wird bald bis auf den letzten Rest ausgetilgt sein. Auch mu $\beta$ man den meisten Bauherren der neuesten Zeit nachsagen, da $\beta$ sie mit Geschmack bauen und nicht lediglich das bessere Zinserträgnis, sondern auch die Schönheit und Harmonie der äußeren Form im Auge behalten. Ästhetiker, obwohl sie selbst gewöhnlich keine Häuser besitzen, wissen dies recht gut zu schätzen und zu würdigen, und auch der Schönheitssinn des größeren Publikums schärft und erhöht sich durch den Anblick eines edlen und geschmackvollen Baustiles. ${ }^{33}$

\footnotetext{
31 Franz Gräffer, „Häuser-Faksimile und noch etwas“ (1845). In: Sehnsucht nach Alt-Wien. Texte zur Stadt, die niemals war. Hrsg. von Arnold Klaffenböck (wie Anm. 1), S. 31-34, hier: S. 31.

$32 \quad$ Ebd., S. 33.

33 Heinrich Joseph Adami, „Neubauten und Wohnungslotterie“ (1847). In: Sehnsucht nach Alt-Wien. Texte zur Stadt, die niemals war. Hrsg. von Arnold Klaffenböck (wie Anm. 1), S. 35-36, hier: S. 35.

Adami - Jurist, Schriftsteller und Journalist - gab 1841-42 die Anthologie Alt- und Neu-Wien. Beiträge zur Beförderung lokaler Interessen für Zeit, Leben, Kunst und Sitte heraus.
} 
Schon um 1800 hob auch nachdrücklich der Schriftsteller, Übersetzer und Bibliothekar Johann Pezzl (1756-1823) in seiner berühmten Skizze von Wien, welche zu gutem Recht als eine der inhaltlich umfassendsten und zuverlässigsten Stadt- und Gesellschaftsbeschreibungen des josephinischen Wien gilt, die vielen Verschönerungen hervor, die Wien erlebt hätte: „Die Stadt selbst ist ungemein dadurch verschönert worden, da $\beta$ manche der alten finsteren Klöster und viele rußige, ungestalte, düstere Höfe und Häuser niedergerissen, und ihre Stellen mit neuen, lichten, bequemen Gebäuden besetzt worden sind.“ $(1790)^{34}$

Eines der auffälligsten Beispiele in der österreichischen Literatur für den (vermeintlichen) Antagonismus zwischen Alt- und Neu-Wien bildet die Heraufbeschwörung der angeblichen Rivalität zwischen den zwei berühmtesten Vertretern des „Wiener Volkstheaters“ im 19. Jahrhundert: Ferdinand Raimund (1790-1836), als Symbol für AltWien, und Johann Nestroy (1801-1862), der mit Neu-Wien assoziiert wird. ${ }^{35}$ Viele Autoren präsentieren die Theater- und Zeitenwende, die in ihren Augen mit Nestroy stattfindet, sowohl als einen ästhetischen Paradigmenwechsel wie auch als einen schmerzlichen Bruch mit der von Ferdinand Raimund und Alt-Wien verkörperten Tradition. ${ }^{36}$ Zum Beispiel greift Ann Tizia Leitich (1891-1976) auf das Oppositionspaar Raimund-Nestroy zurück, um die Widersprüche ihrer Zeit zu erläutern und ihre Sicht einer Spaltung innerhalb des Biedermeiers $\mathrm{zu}$ bestätigen. Leitich räumt deutlich Raimund den Vorzug ein und integriert ihn in die „Oberseite“ des Biedermeiers, während sie Nestroy in dessen „Unterseite“ einreiht. ${ }^{37}$ Diese Tendenz zur Polarisierung zwischen Raimund und Nestroy - als Opposition zwischen Altund Neu-Wien - wird noch spürbarer in der Unterhaltungsliteratur um 1900, etwa bei Karl Hans Strobl (1877-1946), Fritz Stüber-Gunther (1872-1922) oder Hertha Pauli (1906-1973), die allesamt aus Raimund das Symbol für einen begabten, aber im Alltag selbstzweifelnden, zerrissenen und (sowohl beruflich als auch privat) desillusionierten Künstler machen, welcher im Gegensatz steht zu dem unbändigen Freiheitsdrang und den unmoralischen Bestrebungen Nestroys, der sich gegen eine Umwelt auflehnt, die ihn zum „exzentrische[n] Sonderling“‘38 stemple. In Strobls Erzählung Ferdinand Raimund. 10 September 1835 (1922) wird das Bild

\footnotetext{
$34 \quad$ Zit. in Alt-Wien. Die Stadt, die niemals war (wie Anm. 1), S. 11.

35 Zur immer polemischen und oft schematischen Opposition zwischen dem „edlen Moralisten“ Raimund und dem „,kühlen Berechner“ Nestroy vgl. u.a. Young-Kyun Ra, „,Warme Kunst“ versus ,kalte Technik“ Raimund versus Nestroy“. Nestroyana, 24 (2004), Heft 3-4, S. 143-147.

$36 \quad$ Vgl. Klaffenböck (wie Anm. 4), S. 161.

$37 \quad$ Vgl. Leitich (wie Anm. 24), S. 75.

Hertha Pauli, Toni. Ein Frauenleben für Ferdinand Raimund. Roman. Wien: Paul Zsolnay Verlag 1936, S. 31 .
} 
eines intakten Alt-Wien sogar in Raimunds „Schlafkammer“ angesiedelt, wobei der aggressive Ton, mit dem Nestroy in Der böse Geist Lumpacivagabundus (1833) die gesellschaftlichen Mängel und Missstände bloßstellt, die feine und idealistische Poesie (Schiller'scher Prägung) Raimunds zerstöre:

Eine possenhafte Geistergesellschaft verhöhnte Raimunds hochgemute, sittlich ernste Überwelt. Leim, Zwirn und Knieriem zogen das große Los und begannen ihre Irrfahrt, die alle Angelegenheiten zwischen den Menschen und den Mächten über den Menschen wie in Hohlspiegeln brach und verzerrte. Erbittert war Raimund dem Spiel gefolgt; als aber, immer saftiger und urwüchsiger, in all dem Treiben und ein strahlend-boshafter Witz sich entfaltete, der jegliches Ziel wie mit einer Wurflanze traf und jegliche Aufgeblasenheit zerplatzen machte, da begann er den Kopf zu schütteln. $[\ldots]$

Je weiter das Spiel fortschritt, desto deutlicher wurde es Raimund, da $\beta$ hier an die Stelle seiner in phantastischen Sphären heimischen Gewalten, die sich mit den Menschen in ernsthaftem Ringen maßen und ihnen Prüfungen und Aufgaben hohen Wertes stellten, eine lockere, aber überaus vergnügliche Taschenspielerei gesetzt war. $^{39}$

Zusammenfassend wird Raimund zur Inkarnation der Seele und Authentizität AltWiens erhoben, während Nestroy als dessen brutaler Zerstörer oder Totengräber im Bereich des Volkstheaters dargestellt wird. Jene Idee, die u.a. in Stüber-Gunthers Rappelkopf zum Ausdruck kommt, ist auch in Hertha Paulis Erstlingsroman Toni (1936) anzutreffen, wo Raimund seine hohen künstlerischen Ansprüche und Ideale durch Nestroy „verraten“ sieht. Hinzu kommt, dass Nestroys Pragmatismus und Respektlosigkeit gegenüber den Kunstgesetzen die ethisch-künstlerische Strenge Raimunds untergraben hätten: „Wie kam dieser Nestroy dem Publikumsgeschmack entgegen! Und wie hatte er, Raimund, sich bemüht, sein Publikum zu erziehen, er hatte alles Hohe verehrt, nun wurde es vom lästernden Nestroy mit Füßen getreten. Statt des Zauberstabes der Phantasie schwang er die Geißel der Satire. Das war das Ende der Romantik." 40 Der polemische Gegensatz Raimund-Nestroy, der bis in die Mitte des 20. Jahrhunderts fortbestand, diente dazu, beide Autoren, in denen man entgegengesetzte (künstlerische, theatralische, ja weltanschauliche) Prinzipien zu erkennen glaubte, zu etikettieren und gegeneinander auszuspielen. Wie hier kurz präsentiert, war dies

39 Zit. nach der leicht veränderten Neuauflage: Karl Hans Strobl, Ein Schicksalstag Ferdinand Raimunds. Novelle, Wien, Leipzig: Wiener Verlagsgesellschaft 1940, S. 47-49.

$40 \quad$ Pauli (wie Anm. 38), S. 282. 
ein beliebter Topos in der Belletristik, in populärwissenschaftlichen Darstellungen zum Biedermeier und sogar teilweise in der Literaturgeschichtsschreibung. ${ }^{41}$

Schließlich lässt sich ein letztes Beispiel für diesen Riss innerhalb des anscheinend idyllischen Alt-Wien-Mythos anführen. Für Hermann Bahr (1863-1934), Wortführer des „Jungen Wien“ im Café Griensteidl, Theoretiker des Impressionismus und Förderer des Naturalismus und Expressionismus, gehört Alt-Wien unbestreitbar der Vergangenheit an: „Was wird nun aus Wien? Wird überhaupt noch was? Ist es noch fähig zur Zukunft? Zu irgend einer Zukunft? Hat es noch eine Möglichkeit? Viele zweifeln. Und sie sind gar nicht traurig. Sie sagen: Täuschen wir uns nicht, wir haben ausgespielt, wir sind vorbei, wir sind Geschichte, wir sind eine schöne Erinnerung! [...] $]^{\text {42 }}$ In seinen Schriften - insbesondere in seinen Skizzen und Tagebüchern - zeigt sich Bahr regelmäßig kritisch gegenüber einer Stadt, die er immer wieder zu einer „,barocke[n] Schauwand“433 herabsetzt, und greift insbesondere die „Neigung“ der Wiener an, jeglichen Fortschritt abzulehnen. Im Allgemeinen verwirft Bahr die massive Apologie Alt-Wiens in der Literatur, weil diese dazu führe, die aktuelle Situation und die Realität der Stadt aus den Augen zu verlieren, ja sie bewusst zu ignorieren. ${ }^{44}$

Als um 1910 in den Feuilletons wieder einmal ein besonders heftiger Kampf zwischen den „Alt-Wienern“, die sich über das Verschwinden ihrer vertrauten Gassen und Vorstadthäuser beschwerten, und den Modernisten tobte, meldete sich der Satiriker Karl Kraus in der Fackel zu Wort und spielte die beiden Lager gegeneinander aus, indem er dem Märchen von „Alt-Wien“ mit einer sarkastischen Bemerkung definitiv allen Zauber und alle Sentimentalität nahm: „Ich muß den Ästheten eine niederschmetternde Mitteilung machen: Alt-Wien war einmal neu. “45

Abschließend sei anzumerken, dass die utopischen Inhalte und Komponenten, die im Mythos „Alt-Wien“ enthalten sind, vor allem folgende Funktionen erfüllen: Flucht/Ausflucht, Sublimierung/Idealisierung, Schöpfung eines utopischen und bequemen „Unorts“ mit stark

41 Vgl. u.a. Martin Stern, „Die Nestroy-Polemik des deutschen Vormärz - Vorspiel des ,poetischen Realismus ““. In: Johann Nepomuk Nestroy. Tradizione e trasgressione. Hrsg. von Gabriella Rovagnati. Mailand: CUEM 2002, S. 43-60.

42 Hermann Bahr, „Die Zukunft Wiens“ (1906). In: Sehnsucht nach Alt-Wien. Texte zur Stadt, die niemals war. Hrsg. von Arnold Klaffenböck (wie Anm. 1), S. 89-91, hier: S. 89.

$43 \quad$ Hermann Bahr, Schwarzgelb. Berlin: Fischer 1917, S. 51: „barocke Schauwand“. Diese Angriffe von Bahr auf Alt-Wien sind auch in seinem Roman Die Rotte Korahs (1919) zu finden.

${ }_{44}$ Vgl. Hermann Bahr, Tagebuch 1, Eintrag vom 1. Januar 1908. In: H. B., Tagebücher, Skizzenbücher, Notizhefte (Band 5: 1906-1908). Hrsg. von Moritz Csáky. Wien, Köln, Weimar: Böhlau 2003, S. 367.

$45 \quad$ Karl Kraus, Aphorismen. In: K. K., Schriften (Band 8). Hrsg. von Christian Wagenknecht (wie Anm. 29), S. 257. Diesen Aphorismus veröffentlichte Kraus zuerst in der Fackel und dann in dem Band Pro domo et mundo. Insgesamt fühlte sich Kraus von Alt-Wien-Elegien (Werner Sombart, Felix Salten) ,gelangweilt“" und betonte gegenüber Nostalgikern gerne, wie sehr er die Funktionalität moderner Städte schätze. 
konservativen Zügen. Die vielfältige Literatur zu Alt-Wien ersetzt immer wieder die Realität der modernen Stadt durch eine andere - zum großen Teil erdachte, wunderbare und utopische - Welt, durch eine imaginäre Realitätsebene, die sich deutlich von der urbanen Situation Wiens um 1900 unterscheidet, ja in einem krassen Gegensatz zu dieser steht. ${ }^{46}$

In der österreichischen Literatur der Jahre 1850 bis 1930 bildet Alt-Wien sowohl einen selbständigen imaginären Raum als auch einen ungenauen, ewigen urbanen Raum, d.h. eine ästhetische Phantasmagorie. Insofern scheint Alt-Wien in der Literatur durchaus dem Unort im Sinne des französischen Ethnologen und Anthropologen Marc Augé zu entsprechen: „Der Raum des Unorts schafft weder eine besondere Identität noch Beziehungen, sondern Einsamkeit und Ähnlichkeit ${ }^{47}$.“

\footnotetext{
$46 \quad$ Vgl. Marion Linhardt, Residenzstadt und Metropole. Zu einer kulturellen Topographie des Wiener Unterhaltungstheaters (1858-1918). Tübingen: Niemeyer 2006, S. 262.

$47 \quad$ Marc Augé, Non-lieux. Introduction à une anthropologie de la surmodernité. Paris: Seuil 1992, S. 130: „L'espace du non-lieu ne crée ni identité singulière, ni relation, mais solitude et similitude.“
} 